Revisão / Review

\title{
Deficiência de ferro nas afecções gastrointestinais da criança
}

Iron deficiency and the intestinal tract in children

Mauro B. Morais

\section{Introdução}

Deficiência de ferro constitui, na atualidade, a principal carência nutricional na população mundial. ${ }^{1}$ A faixa etária pediátrica inclui dois grupos de risco em fases da vida com maior velocidade fisiológica de crescimento: lactentes e adolescentes. Mulheres na fase reprodutiva e gestantes são os dois outros grupos mais suscetíveis. Adeficiência de ferro é o resultado do balanço negativo imposto por necessidades de ferro que ultrapassam sua biodisponibilidade na dieta. ${ }^{1}$

Uma das principais reações do organismo frente ao balanço negativo de ferro é o aumento da absorção intestinal do ferro da dieta como uma tentativa de evitar a instalação da deficiência de ferro. ${ }^{2}$ É evidente que este mecanismo apresenta eficiência limitada, dependendo da quantidade e tipo de ferro na dieta e da magnitude do fator determinante do balanço negativo. Assim, o intestino tem um papel fundamental na regulação do ferro corporal, especialmente nas situações de carência deste mineral. Mais recentemente, a identificação do hormônio hepcidina, produzido no fígado, confirma a relevante participação do aparelho digestivo na regulação do metabolismo do ferro. ${ }^{3}$

Há mais de 20 anos consolidou-se o entendimento de que a deficiência de ferro é uma condição patológica sistêmica que provoca repercussões que se somam à anemia ferropriva, entre as quais as anormalidades imunológicas, que modificam a susceptibilidade às infecções, alterações no metabolismo, déficit de crescimento, retardo no desenvolvimento neuro-

Gastroenterologista pediatra. Chefe do Departamento de Pediatria da Universidade Federal de São Paulo, Unifesp - São Paulo-SP.

Disciplina de Gastroenterologia Pediátrica do Departamento de Pediatria da Universidade Federal de São Paulo - São Paulo-SP.

Correspondência: Mauro Batista de Morais

Rua dos Otonis, 880 apto 63

04025-901 - São Paulo-SP - Brasil

E-mail:mbmorais@osite.com.br

Doi: 10.1590/S1516-84842010005000061 
psicomotor e, também, alterações na morfologia e fisiologia intestinal. ${ }^{4}$

Assim, a relação entre o ferro e o intestino pode ser analisada sob várias perspectivas: intestino como sede da absorção do ferro regulada pela quantidade de ferro corporal, incluindo a ação da hepcidina produzida no fígado, interação do ferro com outros nutrientes, repercussões da deficiência de ferro no intestino e o intestino como sede de perdas patológicas que podem causar ou agravar a deficiência de ferro. O objetivo deste artigo é abordar estes aspectos da interação entre o ferro e intestino.

\section{Repercussões da deficiência de ferro no intestino}

Este tópico pode ser analisado com base em dados obtidos em estudos com humanos ou com animais de experimentação.

$\mathrm{Na}$ década de 1960, evidenciou-se atrofia parcial das vilosidades intestinais em crianças com anemia ferropriva grave. ${ }^{5,6}$ Estes resultados foram confirmados indiretamente por meio da análise da permeabilidade intestinal em crianças israelenses e guatemaltecas. ${ }^{7,8}$ Assim, surgiram preocupações de que a anemia ferropriva grave poderia, em função destas anormalidades no intestino delgado, provocar redução da absorção não somente dos diferentes macronutrientes da dieta como também reduzir a capacidade de absorção de ferro e, assim, reduzir a eficácia da ferroterapia oral. ${ }^{9}$ Algumas evidências neste sentido foram obtidas com a realização do teste de elevação do ferro sérico após administração de uma dose padrão de ferro (sulfato ferroso) para crianças com anemia ferropriva grave. O objetivo deste teste é avaliar a capacidade absortiva de ferro pelo indivíduo, ${ }^{9}$ no entanto, não tem indicação para aferir a absorção do ferro de alimentos que pode ser feita com o emprego do isótopos estáveis ou radioativos de ferro.

Por outro lado, experimentos com ratos mostraram uma reação contrária no intestino frente à anemia ferropriva provocada por ração com baixo teor de ferro. Assim, observamos diminuição na relação lactulose e manitol na urina de ratos com anemia ferropriva (média de hemoglobina $=$ $11,2 \pm 1,4 \mathrm{~g} ; \mathrm{dL}$ ) em relação aos controles (média de hemoglobina $=16,9 \pm 0,8 \mathrm{~g} ; \mathrm{dL}) .{ }^{10}$ Vale lembrar que as lesões intestinais com atrofia vilositária ocasionam aumento da relação lactulose/manitol por aumento da passagem da lactulose pelos espaços intercelulares e por redução da absorção do manitol por diminuição da superfície absortiva. ${ }^{10}$ No Brasil, com o mesmo modelo animal de anemia ferropriva foi demonstrado, por meio da morfometria do intestino delgado, aumento em cerca de $20 \%$ na altura das vilosidades intestinais e na superfície epitelial, compatível com o aumento na relação lactulose/manitol constatado anteriormente. ${ }^{11}$ Este resultado experimental ${ }^{11}$ difere do encontrado em humanos nos quais pode-se supor que exista a concomitância de dois fatores influenciando inversamente na estrutura do intestino delgado: 1) deficiência de ferro por carência e 2) reação do intestino às condições ambientais insatisfatórias observadas na enteropatia tropical (ambiental). ${ }^{8}$ Neste sentido, crianças com anemia ferropriva podem apresentar anormalidades intestinais secundárias a enteropatia tropical que não permitiriam o aumento da superfície epitelial resultante da deficiência de ferro. Resultados recentes confirmaram que crianças em idade escolar com anemia ferropriva, moradoras em um bolsão de pobreza (favela), apresentam menor absorção intestinal da D-xilose, aferida por meio da D-xilosúria de cinco horas, em comparação com crianças moradoras na mesma favela e que não apresentavam anemia ferropriva. ${ }^{12}$ Este projeto em crianças escolares sem sintomas gastrintestinais reafirma a associação entre anormalidade na função absortiva e anemia ferropriva. ${ }^{12}$ Frente a estas possíveis anormalidades na morfologia e fisiologia intestinal na vigência da anemia ferropriva, conforme já referido, poderia gerar a preocupação de que a absorção de ferro também poderia estar comprometida. Neste contexto, em 1992, realizamos um estudo com crianças atendidas em um serviço público de saúde para avaliar a capacidade da absorção intestinal de ferro na deficiência de ferro. ${ }^{2}$ Foi utilizado o teste da elevação do ferro sérico após a administração, por via oral, de $1 \mathrm{mg} / \mathrm{kg}$ de ferro elementar. Na deficiência de ferro constatou-se aumento na capacidade de absorção do ferro e resposta positiva à ferroterapia oral, demonstrando que a má absorção de ferro não constitui um problema em crianças com anemia ferropriva não associada à outras patologias. ${ }^{2}$

Outra consequência da deficiência de ferro no intestino é a redução na produção de lactase e absorção da lactose. Experimento desenvolvido no Brasil evidenciou menor concentração de lactase no intestino delgado de ratos com anemia ferropriva. ${ }^{13}$ Em crianças pré-escolares assintomáticas constatou-se associação entre diminuição das reservas corporais de ferro e má absorção da lactose. ${ }^{14}$ Deve ser ressaltado, no entanto, que, apesar da má absorção de lactose indicada pelo teste do hidrogênio no ar expirado, não se constatou intolerância à lactose ou menor consumo de leite por estas crianças em idade pré-escolar.

Ainda, no campo das repercussões da anemia ferropriva no intestino delgado, em nosso laboratório observou-se menor absorção de glicose e sódio em ratos com anemia ferropriva com o emprego de um modelo de perfusão de alça do intestino delgado com solução de reidratação oral. É interessante mencionar que esta anormalidade acompanhouse de aumento do conteúdo relativo de RNA mensageiro do cotransportador de glicose (SGLT1). ${ }^{15}$

\section{Influência das fibras alimentares na absorção intestinal de ferro}

Parcela dos profissionais da saúde acredita que as fibras alimentares podem reduzir a absorção de minerais, incluindo o ferro. Outro efeito fisiológico atribuído às fibras 
alimentares é ocasionar saciedade precoce. ${ }^{16}$ Em função destes dois supostos efeitos, existe preocupação de administrar fibras alimentares em excesso para lactentes, o que poderia reduzir o crescimento em função da saciedade precoce e do aumento do risco de anemia por deficiência de ferro, considerando a possibilidade de sua absorção no intestino. Por este motivo, ao que tudo indica, ainda não existe recomendação para consumo mínimo de fibras alimentares no primeiro ano de vida. Por outro lado, constipação intestinal com frequência tem início no primeiro ano de vida e a sua prevenção poderia ser conseguida pela utilização de alimentação rica em fibras a partir da introdução, na dieta, de alimentos complementares ao leite. ${ }^{17,18}$ Vale ressaltar que, no leite materno, são encontrados carboidratos não absorvíveis, denominados oligossacarídeos, que apresentam função prebiótica. ${ }^{19}$ A função prebiótica é caracterizada pela capacidade de parcela destes carboidratos do leite humano estimular o crescimento de bifidobactérias, microorganismos considerados benéficos na microbiota intestinal. ${ }^{19}$ Mais recentemente, certas misturas de prebióticos foram adicionadas às fórmulas prescritas para lactentes que deixaram de ser amamentados de forma natural. ${ }^{20,21}$ Neste contexto, deve ser lembrado que parcela dos prebióticos apresenta características similares às fibras solúveis ou muito fermentáveis.

Considerando as dificuldades para realizar pesquisas em lactentes a respeito do provável efeito inibitório dos diferentes tipos de fibra na absorção intestinal de ferro e no crescimento, foi elaborado, em nossa instituição, um modelo experimental com ratos recém-desmamados..$^{22}$ Ratos com 21 dias de vida foram alimentados com ração padrão sem a adição de ferro (mistura mineral sem ferro) pelo período de duas semanas. Nesta etapa, os animais desenvolvem anemia ferropriva com hemoglobina inferior a 7,0 g/dL. Na segunda etapa, para avaliação do efeito da fibra na absorção de ferro e no crescimento, os animais são alimentados com ração com teor pré-estabelecido de ferro (30 mg por quilo de ração) pelo período de três semanas. Nesta fase, mensura-se a recuperação da hemoglobina, a ingestão alimentar e o ganho de peso. No último dia do experimento, é obtida uma amostra de tecido hepático para determinação do teor de ferro como indicação dos reservas de ferro. No primeiro estudo desta linha constatou-se que a celulose não apresenta efeito negativo sobre a absorção intestinal de ferro ou sobre o crescimento. ${ }^{22}$ Em um segundo experimento, verificou-se que farelo de trigo, usado com freqüência como fonte de fibra insolúvel, também não reduz a absorção de ferro ou o crescimento. ${ }^{23}$ Por sua vez, a goma guar parcialmente hidrolisada determinou aumento na absorção intestinal de ferro, aferida não somente pela regeneração da massa hemoglobínica como também pelo balanço entre a quantidade de ferro ingerido e eliminado nas fezes. ${ }^{23}$ Estudou-se, também, o efeito da pectina que se associou com pequena restrição na ingestão de ração e ganho de peso, sem comprometer a eficiência alimentar ou a absorção intestinal de ferro. ${ }^{24} \mathrm{Os}$ prebióticos inulina e frutooligossacarídeos ocasionaram aumento na absorção intestinal de ferro sem efeito negativo na ingestão de ração e ganho de peso. ${ }^{25}$ Finalmente, avaliouse o efeito de uma mistura de fibras insolúveis e solúveis, incluindo prebióticos, não se constatando interferência na absorção de ferro, ingestão alimentar e ganho de peso. ${ }^{26}$ Assim, nenhuma das fibras analisadas reduziu a absorção intestinal de ferro, sendo que a goma guar parcialmente hidrolisada, inulina e futooligossacarídeos determinaram aumento na absorção de ferro. Apenas a pectina acompanhou-se de menor ganho de peso atrelado à menor ingestão de ração.

\section{Anemia em afecções do aparelho digestório}

A principal causa de anemia por deficiência de ferro é carencial. No entanto, afecções do aparelho digestivo podem ocasionar ou agravar o déficit corporal de ferro, basicamente, por reduzir a absorção do ferro ou por aumentar as perdas de ferro pelo intestino.

Na infância e adolescência, quanto à perda de sangue a partir do esôfago, a única menção existente relaciona-se à esofagite por refluxo. Pela erosão da mucosa pode ocorrer sangramento. Entretanto, na prática, a anemia ferropriva não constitui um problema clínico de relevância no tratamento da esofagite por refluxo. ${ }^{28}$ Não foram encontradas informações na literatura a respeito de anormalidades nos compartimentos corporais de ferro na esofagite eosinofílica, que é uma entidade com inflamação crônica do esôfago.

Causas de sangramento no estômago vinculam-se, principalmente, às gastrites secundárias ao uso de medicamentos que, potencialmente, dependendo da magnitude da hemorragia, podem ocasionar deficiência de ferro. Outras causas de sangramento digestivo na Pediatria incluem divertículo de Meckel, invaginação intestinal e anomalias vasculares, entre outras malformações. ${ }^{29}$

Existem evidências de que a infecção pelo Helicobacter pylori pode ocasionar repercussões nos compartimentos de ferro corporal. De acordo com uma revisão sistemática, foram publicados na literatura, até o final de 2006, 12 relatos de séries ou casos, 19 estudos populacionais observacionais e seis estudos de intervenção. A metanálise mostrou que indivíduos com infecção por $\mathrm{H}$. pylori apresentam risco de anemia quase três vezes maior do que os não infectados. Risco de deficiência de ferro é 1,8 vezes maior nos infectados. A análise dos dados dos estudos epidemiológicos mostra que as médias da hemoglobina dos infectados e não infectados não são diferentes a ponto de indicar uma grande relevância clínica. O mecanismo de produção da deficiência de ferro não está plenamente esclarecido e sugere-se a participação dos seguintes fatores: 1 . Aumento das perdas sanguíneas gastrintestinais; 2. Diminuição da absorção intestinal de ferro e 3. Captação do ferro pelas bactérias. ${ }^{30}$ 
Como causas de anemia por perda de sangue oculto destacam-se, na faixa etária pediátrica, as parasitoses intestinais e a alergia à proteína do leite de vaca.

Quanto às parasitoses, a ancilostomíase representou, no passado, uma causa muito valorizada de anemia ferropriva. Nos textos de Monteiro Lobato inclui-se a clássica família do Jeca Tatu, acometida pelo "amarelão". Necator americanus e Ancylostoma duodenale são parasitas intestinais hematófagos que provocam perda sanguínea proporcional ao número de parasitas. ${ }^{31}$ Assim, estima-se que a presença de $1.000 \mathrm{ovos} /$ grama de fezes provoque perda sanguínea diária de $2 \mathrm{~mL}$ para o $N$. americanus, $4 \mathrm{~mL}$ para o A. duodenale. Trichiurus trichiura, com habitat no intestino grosso, ocasiona perda sanguínea menor $(0,25 \mathrm{~mL} / \mathrm{dia}){ }^{31}$

Em uma comunidade rural de crianças indígenas da etnia Terena, em Aquidauana (Mato Grosso do Sul), encontrou-se anemia (redução da hemoglobina) em 95,6\% (43/45) dos lactentes, em 85,5\% (59/69) dos pré-escolares e em 48,9\% $(59 / 121)$ dos escolares. ${ }^{32}$ Nesta comunidade, pesquisa realizada anteriormente demonstrou o seguinte perfil de parasitoses intestinais: Giardia lamblia em 30,3\% das crianças, Hymenolepsis nana em 10,6\%, Necator americanus em 3,3\%, Strongyloides stercoralis em 3,3\% e Ascaris lumbricoides em 1,6\%. Portanto, a ocorrência de ancilostomíase em 3,3\% das crianças não poderia explicar as altas taxas de anemia encontradas nestas crianças da etnia Terena. ${ }^{32} \mathrm{Ou}$ seja, ao que tudo indica, a natureza da anemia nesta comunidade Terena é de etiologia ferropriva.

Ainda, no tópico das relações entre parasitoses intestinais e deficiência de ferro, um grupo de pesquisadores italianos, na década de 1980, demonstrou má absorção de ferro em crianças com giardíase sintomática. ${ }^{33}$ Vale lembrar que a Giardia lamblia é um protozoário cujo habitat é o início do intestino delgado, mesmo local onde ocorre a absorção intestinal do ferro. O estudo italiano incluiu crianças com diarreia crônica. ${ }^{33} \mathrm{~A}$ absorção intestinal de ferro foi avaliada com base na elevação do ferro sérico após uma dose de ferro em jejum. ${ }^{33}$ No Brasil, foi avaliada a absorção intestinal de ferro em crianças com anemia ferropriva associada ou não com giardíase assintomática. Ressalte-se que a giardíase assintomática é a forma clínica mais frequente desta parasitose na população pediátrica. Outro aspecto importante é o fato de que as crianças com anemia ferropriva deveriam apresentar aumento da absorção intestinal de ferro. O teste de absorção com base na elevação do ferro sérico mostrou valores muito semelhantes nos dois grupos, portadores ou não de giardíase. Os dois grupos receberam ferroterapia oral $(5 \mathrm{mg} / \mathrm{kg} / \mathrm{dia})$ na forma de sulfato ferroso e, após trinta dias, apresentaram a mesma elevação da hemoglobina. Portanto, estes resultados mostraram que a giardíase assintomática não provoca redução na absorção de ferro ou comprometimento da eficácia da ferroterapia oral..$^{34}$

A doença celíaca é uma enteropatia ocasionada por intolerância ao glúten em indivíduos suscetíveis. Mecanismo de imunidade celular faz parte da fisiopatalogia da doença, que ocasiona atrofia nas vilosidades do intestino delgado acompanhada de aumento no número de linfócitos intraepiteliais. Portanto, pode provocar má absorção intestinal de ferro. Quanto ao quadro clínico, a doença celíaca pode ser classificada em: 1. clássica, 2. atípica e 3. assintomática. Assim, anemia é uma das manifestações clínicas da forma clássica da doença celíaca onde a diarreia crônica é a principal manifestação, associada com comprometimento do estado nutricional e anemia ferropriva. ${ }^{35}$ Dentre as formas atípicas da doença celíaca inclui-se a anemia ferropriva refratária à ferroterapia oral. Deve ser lembrado, no entanto, que, na prática médica, a ferroterapia oral prescrita ou administrada de forma inadequada é a principal causa de insucesso terapêutico. Deve ser ressaltado que o diagnóstico da doença celíaca deve ser estabelecido, obrigatoriamente, com o emprego de biópsia do intestino delgado. A pesquisa de anticorpos da classe IgA antitransglutaminase e antiendomísio pode ser utilizada na triagem diagnóstica da doença celíaca após exclusão de deficiência de $\operatorname{IgA}$, que pode ocasionar resultados falsos negativos. Estes anticorpos da classe IgA podem ser utilizados na pesquisa ativa de doença celíaca em grupos de risco, como diabete melito, síndrome de Down e parentes de portadores de doença celíaca. ${ }^{35}$ Pesquisa de doença celíaca não previamente diagnosticada entre 300 parentes de pacientes com doença celíaca mostrou que os 18 indivíduos recém-identificados como portadores de doença celíaca (triagem sorológica confirmada por biópsia de intestino delgado) apresentavam valores médios de hemoglobina inferiores aos dos parentes que não apresentavam doença celíaca ${ }^{36}$ Apesar da prevalência de anemia ferropriva ser maior nos recém-diagnosticados $(11,1 \%)$ do que nos parentes sem doença celíaca (2,7\%), o estudo estatístico não revelou diferença estatisticamente significante, provavelmente pelo número relativamente pequeno de indivíduos recém-diagnosticados. É interessante mencionar que apenas 5 dos 18 indivíduos eram completamente assintomáticos enquanto os demais apresentavam sintomas compatíveis com doença celíaca, especialmente diarreia, que não era valorizada como indicador da doença. ${ }^{36}$

Nas doenças hepáticas crônicas é comum ser encontrado anemia. A maior parte dos conhecimentos a respeito de anemia na doença hepática crônica é proveniente de pesquisas com pacientes portadores de cirrose alcoólica. Considera-se que os seguintes fatores estão envolvidos na gênese da anemia no hepatopata: aumento do volume plasmático, hemorragias por varizes de esôfago, hemólise, deficiência de elementos como ferro, vitamina B12 e ácido fólico e anormalidades próprias da inflamação crônica. ${ }^{37} \mathrm{~A}$ anemia da doença hepática crônica, especialmente a colestática, apresenta características compatíveis com a anemia da inflamação crônica na qual pode ser observada diminuição do tempo de vida das hemácias, redução da utilização do ferro sequestrado no sistema retículo-endotelial e, ainda, redução 
na absorção intestinal de ferro. Estas características permitem a inclusão da anemia da doença hepática crônica no grupo da anemia da inflamação crônica.

Considerando que este assunto foi pouco investigado em crianças, foi realizado um estudo em nosso ambulatório com o objetivo de avaliar a ocorrência de anemia e a absorção intestinal de ferro, além do padrão da dieta e a ocorrência de desnutrição energético-proteica em crianças com doença hepática crônica.$^{37}$ Foram estudados 25 pacientes. A idade variou de 6,5 meses a 12,1 anos. Além dos indicadores do ferro corporal, foi realizado teste de absorção do ferro (incremento do ferro sérico duas horas após a ingestão de sulfato ferroso) e teste terapêutico com sulfato ferroso $(5 \mathrm{mg} / \mathrm{kg}$ de ferro elementar) pelo período de trinta dias. Como referencial, foram considerados os valores obtidos no teste de absorção de ferro e a resposta à ferroterapia oral observados em 27 crianças com anemia ferropriva. Neste grupo de portadores de doença hepática crônica, os 14 com doença colestática apresentavam déficit antropométrico mais intenso do que os hepatopatas sem colestase. Anemia foi encontrada em 11 dos 14 dos pacientes com colestase e em 7 dos 11 sem colestase. O grupo com anemia e doença hepática com colestase, que apresentou maiores valores de ferritina, teve menor absorção intestinal de ferro do que os hepatopatas sem colestase e dos portadores de anemia ferropriva sem doença hepática. ${ }^{37}$ Vale ressaltar que todos os grupos apresentaram resposta de elevação da hemoglobina pela ferroterapia oral, sendo os valores médios inversamente proporcionais ao valor inicial da hemoglobina. ${ }^{37}$

Em 2001, foi isolada a hepcidina, hormônio produzido no fígado para regular a absorção intestinal de ferro e a utilização do ferro de macrófagos por sua ação sobre a ferroportina. ${ }^{3}$ Considerando que nas hepatopatias crônicas ocorre redução na função hepática e, com frequência, anemia por deficiência de ferro e/ou pela inflamação, foi realizado um estudo, em nosso ambulatório, no qual se determinou a concentração sérica de prohepcidina em crianças com doença hepática crônica e em um grupo controle constituído por crianças sem doença hepática ou deficiência de ferro. ${ }^{38}$ Constatou-se que, na doença hepática crônica, os níveis de prohepcidina são maiores do que nos controles, indicando uma resposta à inflamação que, no entanto, não se acompanhou de redução na capacidade de absorção intestinal de ferro. Observou-se forte correlação negativa entre os níveis de transferrina e prohepcidina. ${ }^{38}$ Vale ressaltar que, no futuro, novas pesquisas devem quantificar o teor sérico da hepcidina propriamente dita, o que ainda não é possível, por serem disponíveis comercialmente os reagentes necessários para sua determinação.

Para o final deste artigo ficou reservado um assunto da maior relevância: relação entre o tipo de leite utilizado para o lactente e deficiência de ferro. Vale lembrar que lactentes apresentam elevada prevalência de deficiência de ferro e anemia, conforme mostrados por estudos epidemiológicos realizados no Brasil, os quais utilizaram a ferritina sérica em adição à hemoglobina na aferição dos compartimentos corporais de ferro. ${ }^{39,40}$ Deve ser ressaltado que o aleitamento natural exclusivo é adequado para suprir as necessidades de ferro no primeiro semestre de vida, em função da maior biodisponibilidade do ferro no leite humano. A partir do segundo semestre de vida, os lactentes em aleitamento artificial devem receber suplementação de ferro conforme recomendado pela Sociedade Brasileira de Pediatria. ${ }^{41}$ Estudo realizado com lactentes atendidos em uma unidade básica de saúde em Belém, Pará, mostrou que o aleitamento natural exclusivo nos primeiros quatro meses de vida não constituiu-se em fator de proteção contra anemia por deficiência de ferro no segundo semestre e segundo ano de vida. ${ }^{40}$ Por outro lado, antecedente de uso de fórmula infantil fortificada com ferro no momento da interrupção do início do aleitamento natural foi caracterizado como um fator de proteção contra o aparecimento de anemia ferropriva. ${ }^{40}$ Vale ressaltar que o emprego de fórmula infantil fortificada com ferro é a alternativa internacionalmente aceita para suceder o leite materno no primeiro ano de vida. ${ }^{42}$ No entanto, uma pesquisa realizada no Brasil, em 2006, mostrou que apenas $39,8 \%$ das crianças recebiam leite materno exclusivo até o sexto mês de vida. ${ }^{43}$

Para o grande contingente de lactentes vítimas da interrupção prematura do aleitamento materno, a utilização de fórmulas lácteas para lactentes fortificadas com ferro (fórmula infantil) constitui uma das alternativas mais adequadas para a sua nutrição e prevenção da deficiência de ferro. ${ }^{41}$ Entretanto, em razão de fatores culturais e socioeconômicos, o acesso e a disponibilidade dessas fórmulas são reduzidos, determinando que o leite de vaca constitua o alimento substituto mais frequentemente utilizado. ${ }^{44}$ Além do leite de vaca integral não apresentar o mesmo teor de ferro das fórmulas para o lactente, considera-se que o maior teor de cálcio e proteínas pode reduzir a biodisponibilidade do ferro. ${ }^{45}$ Poucos estudos avaliaram o efeito destas variáveis nos compartimentos corporais de ferro. Considerando as restrições éticas, foi desenvolvido, em nossa instituição, estudo com ratos com 21 dias de vida que foram alimentados exclusivamente com leite de vaca, fórmula infantil fortificada com ferro e leite de vaca integral fortificado com o mesmo teor e tipo de ferro utilizado na fortificação da fórmula láctea para lactentes. ${ }^{45}$ No início do experimento, as médias de hemoglobina dos três grupos eram semelhantes a 9,0 g/dL. Após dez dias, a média da hemoglobina do grupo alimentado com fórmula infantil fortificada com ferro foi igual a $12,1 \pm 1,1 \mathrm{~g} / \mathrm{dL}$; no grupo com leite de vaca integral fortificado com ferro, a média da hemoglobina foi igual a 9,6 $6 \pm 1,6 \mathrm{~g} / \mathrm{dL}$, e no grupo que recebeu leite de vaca integral sem ferro, a média foi igual a $6,2 \pm 1,0 \mathrm{~g} / \mathrm{dL}$, sendo as diferenças entre os grupos estatisticamente significantes. Estes dados mostram a contundência do efeito de inibição da absorção do ferro pelo leite de vaca integral. ${ }^{45}$ No estudo foram evidenciadas, também, mudanças quanto à 
ingestão de líquidos, excreção renal de ureia e sódio nos animais alimentados com leite de vaca integral. ${ }^{45}$

Alergia à proteína do leite de vaca pode representar uma forma de perda de sangue nas fezes, mais especificamente na colite eosinofílica ou alérgica. ${ }^{46}$ Nesta condição clínica, lactentes em aleitamento artificial ou natural apresentam sangue nas fezes, em quantidade variável, não associada com outras manifestações clínicas como febre, vômitos ou déficit de ganho de peso. Devem ser descartadas as possibilidades de fissura anal e infecção por agente enteropatogênico invasor. Entretanto, fissura anal pode fazer parte do quadro de proctocolite alérgica. Parcela dos lactentes, na dependência da intensidade e duração das perdas sanguíneas nas fezes, pode desenvolver anemia ferropriva. ${ }^{46} \mathrm{~A}$ colite eosinofílica ocorre principalmente nos primeiros meses de vida quando é mais difícil a interpretação dos valores de hemoglobina e ferritina. Assim, deve ser analisada em cada caso a necessidade de dose de ferro para suplementação ou tratamento. ${ }^{46}$

Outra situação atribuída ao leite de vaca é a perda de sangue oculto nas fezes. Apesar de não existirem evidências que permitam associar esta manifestação com alergia às proteínas do leite de vaca, esta última muitas vezes é responsabilizada pelo processo. A presença de sangue oculto nas fezes ocasionando anemia ferropriva em lactentes alimentados com leite de vaca foi reconhecida há mais de 40 anos. ${ }^{47}$ Entretanto, estudo mais recente questiona a significância clínica da presença de sangue oculto nas fezes, mesmo frente à redução da média de ferritina.$^{48} \mathrm{O}$ fato de apresentarem presença de sangue oculto enquanto alimentados com fórmula infantil pode se associar com menor repercussão nos compartimentos de ferro em função da possibilidade de aumento compensatório na absorção intestinal de ferro. Por sua vez, conforme já mencionado, certos grupos de lactentes continuam sendo alimentados com leite de vaca integral. Em Arapongas, no interior do Paraná, foi mostrado que grande parcela dos lactentes no final do primeiro ano de vida apresentavam anemia. ${ }^{49}$ Presença de sangue oculto nas fezes foi associada com menores valores de ferritina no soro. Assim a presença de sangue oculto nas fezes foi considerada um fator agravante da deficiência de ferro. ${ }^{49}$

Concluindo, o tubo digestivo apresenta grande participação no metabolismo do ferro e o conhecimento dos seus mecanismos fisiológicos e patológicos são de relevância na prevenção e tratamento da deficiência de ferro.

\section{Considerações finais}

O intestino é sede de importantes etapas do metabolismo do ferro. A prevenção da deficiência de ferro deve ter início desde o nascimento com a adoção do aleitamento natural exclusivo. Quando o aleitamento natural não é possível, o lactente deve ser alimentado com fórmula láctea modificada e fortificada com ferro, uma vez que a maior quantidade de cálcio e proteínas do leite de vaca integral reduz a absorção de ferro. Outro aspecto negativo é a possibilidade do leite de vaca provocar perda de sangue oculto nas fezes, que agrava a deficiência de ferro. Diminuição da absorção intestinal de ferro é vista apenas em enteropatias graves que acometem a porção intestinal do intestino delgado, como na doença celíaca, que deve fazer parte do diagnóstico diferencial da anemia ferropriva não responsiva à ferroterapia. Déficit da absorção intestinal de ferro pode ser encontrado nas doenças com inflamação, como ocorre nas doenças hepáticas crônicas com colestase, no entanto, estes pacientes podem responder à ferroterapia oral.

\section{Abstract}

The relationships between iron deficiency, iron metabolism and the intestinal tract function can be analyzed from various perspectives: 1. the intestine as the site of iron absorption regulated by hepcidin produced in the liver; 2. the interaction between iron with other nutrients; 3. repercussions of iron deficiency in the intestine and 4. the intestine as the location of pathological loses that can cause or aggravate iron deficiency. The aim of this article is to cover these aspects of the interaction between iron and the intestinal tract. In iron deficiency, an increase in iron absorption has been observed, which in animals is accompanied by an increase in the height of the intestinal villosities. On the other hand, in humans with iron deficiency anemia, abnormalities of the intestinal physiology, different to those found in the laboratory, can occur. Poor intestinal iron absorption can occur in illnesses such as atrophy of the villosities as occurs in celiac disease and in illnesses associated with inflammation anemia such as cholestatic hepatic diseases. Nowadays blood loses from intestinal parasitosis are not a frequent cause of iron deficiency. Infants who have a high risk of developing iron deficiency anemia should be exclusively breast feed and a supplement of iron must be started from their sixth month of life. The use of cow milk is associated with lower iron absorption and blood loses that can aggravate iron deficiency. Infants who do not receive maternal milk must be fed with an infant formula fortified with iron. Rev. Bras. Hematol. Hemoter. 2010;32(Supl.2):62-69.

Key words: Iron deficiency; iron; dietary; occult blood; anemia.

\section{Referências Bibliográficas}

1. World Health Organization. Worldwide prevalence on anaemia 19932005. Geneva: WHO; 2008.

2. Morais MB, Suzuki HU, Machado NL, Fagundes Neto U. Avaliação de um teste simples de absorção intestinal de ferro na deficiência de ferro. J Ped. 1992;68:48-53.

3. Park $\mathrm{CH}$, Valore EV, Waring AJ, Ganz T. Hepcidin, a urinary antimicrobial peptide synthesized in the liver. J Biol Chem 2001;276(11):7806-10.

4. Prasad AN, Prasad C. Iron deficiency: non-hematological manifestations. Prog Food Nutr Sci. 1991;15(4):255-83.

5. Naiman JL, Oski FA, Diamond LK, Vawter GF, Shwachman H. The gastrointestinal effects of iron-deficiency anemia. Pediatrics. 1964;33:83-99. 
6. Guha DK, Walia BN, Tandon BN, Deo MG, Ghai OP. Small bowel changes in iron-deficiency anaemia of childhood. Arch Dis Child. 1968;43(228):239-44.

7. Berant M, Khourie M, Menzies IS. Effect of iron deficiency on small intestinal permeability in infants and young children. J Ped Gastroenterol Nutr. 1992;14(1):17-20.

8. Goto K, Chew F, Torún B, Peerson JM, Brown KH. Epidemiology of altered intestinal permeability to lactulose and mannitol in Guatemalan infants. J Ped Gastroenterol Nutr. 1999;28(3):282-90.

9. Gross SJ, Stuart MJ, Swender PT, Oski FA. Malabsorption of iron in children with iron deficiency. J Pedriatr. 1976;88(5):795-9.

10. de Morais MB, Lifschitz CH. Intestinal permeability to lactulose and mannitol in growing rats with iron deficiency anemia. Biol Trace Elem Res. 2004;99(1-3):233-40.

11. Wayhs ML, Patrício FS, Amancio OM, Pedroso MZ, Neto UF, Morais MB. Morphological and functional alterations of the intestine of rats with iron-deficiency anemia. Braz J Med Biol Res. 2004;37(11):1631-5.

12. Mello CS, Tahan S, Melli LCFL, Rodrigues MSC, Morais MB. Absorção intestinal de D-xilose e anemia em crianças escolares moradoras em um bolsão de pobreza (favela). Rev Chil Nutr. 2009;36(Supl 2):976.

13. Vieira MR, Galvão LC, Fernandes MI. Relation of the disaccharidases in the small intestine of the rat to the degree of experimentally induced iron-deficiency anemia. Braz J Med Biol Res. 2000; 33(5):539-44

14. Cople-Rodrigues CS. Sobrecrescimento bacteriano no intestino delgado, absorção e tolerância à lactose e deficiência de ferro em pré-escolares de duas creches da cidade do Rio de Janeiro. Tese de doutorado: Universidade Federal de São Paulo, 2001.

15. Wayhs MLC. Transporte transepitelial de glicose, sódio e água e RNA mensageiro dos transportadores de glicose no intestino delgado de ratos com anemia ferropriva. Tese de doutorado: Universidade Federal de São Paulo, 2001.

16. Anderson JW, Baird P, Davis RH Jr., Ferreri S, Knudtson M, Koraym A, et al. Health benefits of dietary fiber. Nutr Rev. 2009;67(4):188205 .

17. Morais MB, Maffei HVL. Constipação intestinal. J Pediatr (Rio J). 2000;76(Supl 2):S147-S156.

18. Medeiros LC, Morais MB, Tahan S, Fukushima E, Motta ME, Fagundes-Neto U. Características clínicas de pacientes pediátricos com constipação crônica de acordo com o grupo etário. Arq Gastroenetrol. 2007;44:340-4.

19. Moro G, Minoli I, Mosca M, Fanaro S, Jelinek J, Stahl B, et al. Dosagerelated bifidogenic effects of galacto- and fructooligosaccharides in formula-fed term infants. J Pediatr Gastroenterol Nutr. 2002;34 (3):291-5.

20. Moro G, Arslanoglu S, Stahl B, Jelinek J, Wahn U, Boehm G. A mixture of prebiotic oligosaccharides reduces the incidence of atopic dermatitis during the first six months of age. Arch Dis Child. 2006;91(10):814-9.

21. Arslanoglu S, Moro GE, Schmitt J, Tandoi L, Rizzardi S, Boehm G. Early dietary intervention with a mixture of prebiotic oligosaccharides reduces the incidence of allergic manifestations and infections during the first two years of life. J Nutr. 2008;138(6):1091-5.

22. Catani M, Amâncio OMS, Fagundes-Neto U, Morais MB. Dietary cellulose has no effect on the regeneration of hemoglobin in growing rats with iron deficiency anemia. Braz J Med Biol Res. 2003;36(6):693-7.

23. Morais MB, Catani M, Amancio OMS, Fagundes-Neto U. Wheat bran has no effect on the regeneration of hemoglobin and weight gain in growing rats with iron deficiency anemia. In:
Gastroenterology - Reports from the Second World Congress of Pediatric Gastroenterology (International Proceedings). Medimond, Monduzzi Editore, Bologna, 2004. p. 153-56.

24. de Cássia Freitas K, Amancio OM, Ferreira Novo N, FagundesNeto U, de Morais MB. Partially hydrolyzed guar gum increases intestinal absorption of iron in growing rats with iron deficiency anemia. Clin Nutr. 2006;25(5):851-8.

25. Feltrin C, Batista de Morais M, de Cássia Freitas K, Beninga de Morais T, Fagundes Neto U, Silvério Amancio OM. Effect of soluble fiber pectin on growth and intestinal iron absorption in rats during recovery from iron deficiency anemia. Biol Trace Elem Res. 2009;129(1-3):221-8.

26. Freitas KCL. Efeito dos prebióticos inulina HP e oligofrutose sobre a absorção intestinal de ferro e morfometria do duodeno e cólon de ratos em fase de crescimento. Tese de Doutorado: Universidade Federal de São Paulo, 2009.

27. Weber TK, Freitas KC, Amancio OMS, Morais MB. The fiber mixture of dietary fiber does not affect growth and intestinal iron absorption in growing rats with iron deficiency anemia. In: 4th European Paediatric GI Motility meeting; 2007; London, United Kingdom. Anais. London: 2007. p.84.

28. Vandenplas Y, Rudolph CD, Di Lorenzo C, Hassall E, Liptak G, Mazur L, et al. Pediatric gastroesophageal reflux clinical practice guidelines: joint recommendations of the North American Society for Pediatric Gastroenterology, Hepatology, and Nutrition (NASPGHAN) and the European Society for Pediatric Gastroenterology, Hepatology, and Nutrition (ESPGHAN). J Pediatr Gastroenterol Nutr. 2009;49(4):498-547.

29. Fox VL. Gastrointestinal bleeding in infancy and childhood. Gastroenterol Clin North Am. 2000;29(1):37-66.

30. Muhsen K, Cohen D. Helicobacter pylori infection and iron stores: a systematic review and meta-analysis. Helicobacter. 2008;13 (5):323-40.

31. Olivares M, Walter T, Hertrampf E, Pizarro F. Anaemia and iron deficiency disease in children. Br Med Bull. 1999;55(3):534-43.

32. Morais MB, Alves GM, Fagundes-Neto U. Estado nutricional de crianças índias terenas: evolução do peso e estatura e prevalência atual de anemia. J Pediatr (Rio J). 2005;81:383-9.

33. De Vizia B, Poggi V, Cucchiara S, Acampora A. Iron malabsorption in giardiasis. J Pediatr. 1985;107(1):75-8.

34. De Morais MB, Suzuki HU, Corral JN, Machado NL, Neto UF. Asymptomatic giardiasis does not affect iron absorption in children with iron deficiency anemia. J Am Coll Nutr 1996;15(5):434-38.

35. Sdepanian VL, de Morais MB, Fagundes Neto U. Doença celíaca: a evolução dos conhecimentos desde sua centenária descrição original até os dias atuais. Arq Gastroenterol. 1999;36:244-57.

36. Laranjeira MS. Prevalência de doença celíaca em parentes de primeiro grau de pacientes com doença celíaca em São Paulo. Tese de Mestrado: Universidade Federal de São Paulo, 2005.

37. Mattar RH, Azevedo RA, Speridião PG, Fagundes Neto U, Morais MB. Estado nutricional e absorção intestinal de ferro em crianças com doença hepática crônica com e sem colestase. J Pediatr (Rio J). 2005;81(4):317-24.

38. Basso LDS. Pro-hepcidina sérica, absorção intestinal de ferro e indicadores do ferro corporal em pacientes pediátricos com doença hepática crônica. Rev Chil Nutr. 2009;36(Sup12):959.

39. Hadler MC, Juliano Y, Sigulem DM. Anemia do lactente: etiologia e prevalência. J Pediatr (Rio J). 2002;78(4):321-6.

40. Neves MB, da Silva EM, de Morais MB. Prevalência e fatores associados à deficiência de ferro em lactentes atendidos em um centro de saúde-escola em Belém, Pará, Brasil. Cad Saúde Pública. 2005;21:1911-8. 
41. Morais MB, Sigulem DM. Cows milk, infant formula and irondeficiency anemia. J Pediatr (Rio J). 2006;82:161-2.

42. American Academy of Pediatrics. Committee on Nutrition: The use of whole cows milk in infancy. Pediatrics. 1992;89:1105-9.

43. Brasil. Ministério da Saúde. Secretaria de Ciência, Tecnologia e Insumos Estratégicos. Departamento de Ciência e Tecnologia. PNDS 2006. Pesquisa nacional de demografia e saúde da criança e da mulher. Brasília (DF): Ministério da Saúde; 2008.

44. Assis AMO, Gaudenzi EN, Gomes G, Ribeiro RC, Szarfac SC, Souza SB. Hemoglobin concentration, breastfeeding and complementary feeding in the first year of life. Rev Saúde Pública. 2004;38:543-51.

45. Costa ML, Freitas K de C, Amancio OM, Paes AT, da Silva SM, Luz $\mathrm{J}$, et al. Iron absorption from infant formula and iron-fortified cow's milk: experimental model in weanling rats. J Pediatr (Rio J). 2009;85(5):449-54.

46. Toporovski MS, Vieira MC, Spolidoro JVN, Morais MB, FagundesNeto U. Alergia ao leite de vaca. In: Lopez FA, Campos Jr D. Tratado de Pediatria - Sociedade Brasileira de Pediatria. Segunda Edição. Barueri: Manole; 2010. p. 953-62.

47. Wilson JF, Heiner DC, Lahey ME. Milk-induced gastrointestinal bleeding in infants with hypochromic microcytic anemia. JAMA. 1964;189:568-72.

48. Jiang T, Jeter JM, Nelson SE, Ziegler EE. Intestinal blood loss during cow milk feeding in older infants: quantitative measurements. Arch Pediatr Adolesc Med. 2000;154(7):673-8.

49. Fernandes SMR, Morais MB, Amancio OMS. Intestinal blood loss as an aggravating factor of iron deficiency in infants aged 9 to 12 months fed whole cow's milk. J Clin Gastroenterol. 2008;42 (2):152-6.

O tema foi sugerido e avaliado pelo coeditor deste fascículo educativo, Rodolfo Delfini Cançado, e pelo board interno da RBHH, e publicado após a concordância do editor, Milton Artur Ruiz.

Conflito de interesse: sem conflito de interesse

Recebido: 21/12/2009

Aceito: $16 / 01 / 2010$ 